\title{
Postural stability of human body gazing at moving pictures
}

\author{
Hajime Takada and Motoki Ohba \\ *Department of Mechanical Engineering and Materials Science, \\ Yokohama National University, \\ 79-5 Tokiwadai, Hodogaya-ku, Yokohama 240, Japan \\ ** Komatsu Co. Ltd., 2-3-6, Akasaka, Minato-ku, Tokyo, 107 Japan \\ (Received 16 December 1996)
}

\begin{abstract}
This paper is concerned with movements and stability of man gazing at a moving picture in a darkroom. These experiments studied to clarify the relation between visual information and postures. A worker may indirectly be exposed vibration when the objects around him swing. This is not only a problem for workers but also when he operates a large plant, it is a severe problem for the system if his posture swings and makes a mistake. The bar picture a subject gazes at was made with a computer in this experiments. In the first case, the center of gravity of a subject was measured when the bar swings right and left in front of him and the equilibrium line deviates gradually right or left. In the second case, the effects of gazing for a long time on subject's posture were measured. As results, our center of gravity deviates in the same direction as the equilibrium line deviates. And almost all subjects tune to the swinging bar in $5 \mathrm{~min}$ after they start their examinations, that is, this time may be the limit we can control our posture when we are gazing at a swinging bar in a darkroom.
\end{abstract}

Keywords: Posture of man, Seeing mechanism, Human interface, Working conditions, Moving pictures

PACS number: 43. 40. Ng, 43. 60. Lq

\section{INTRODUCTION}

Some people work under severe conditions, for example with a vibrating tool like a chain saw. Two cases of receiving vibration can be considered. One is the case we receive vibration directly such as from a vibrating tool or from a vibration floor like a seismic condition. The other is the case we receive it indirectly when we are gazing at a moving picture or when we are listening to a swinging sound. It is said that we get a great part of informations from external world through our eyes. When a worker operates a large plant and he make a mistake by swinging his posture, this is not only a problem for workers but also it is a severe problem for the system. This fact is concerned with a human operating error in an emergency because he operates reflexly according to the influence of informations received. In a plant when an automatic control breaks down because of something like an earthquake and man has to operate manually under a vibrating condition or environment, a severe accident may occur if man operates by mistake. When man operates under the condition of his body sway, it is estimated that the probability of an error is higher than that under the normal condition. The information we received may not be true in an emergency. For example, when in a train we are gazing at another starting train, some people think our train is starting, and the others think another train is going. It is very important which imagination we have. If we think another train begins to start when our train is starting, we lose our balance. Even in the opposite case; if we think our train 
begins to start when another train is going, we may lose our balance. There are some papers ${ }^{1-3)}$ on eye movements or vision, but few papers ${ }^{4)}$ on human body vibration in gazing at a moving object. In this paper, we consider how we can stably stand when we are gazing at a moving picture. We examined whether the human responses in a darkroom could be measured using the method we proposed or not. In the experiment, movements of a subject's center of gravity (C. G.) were measured when he standing on a force plate in a darkroom is gazing at a swinging picture made by a personal computer. We had two kinds of cases. In the first case, the C. G. movements of a subject were measured when a bar picture was swinging right and left in front of him, deviating the equilibrium line of the bar vibration, and in the second case the C. G. movements were measured when he was gazing at a moving picture in a darkroom for a long time (11 $\min$ ).

This study makes it clear that the operation efficiency and influences on a human body under the bad labor environment. We hope the results obtained from these experiments on a human body will be used to determine parameters of human vibrating model, by which a good environment or a good man-machine interface will be designed.

\section{EXPERIMENTS}

The movement of man's C. G. were measured with four load transducers under the force plate in a darkroom as shown in Fig. 1. A subject puts on a pair of sun-glasses so that he cannot see any objects other than the picture moving in the CRT display. As shown in Fig. 2, the picture is rectangular and its size is $12 \mathrm{~mm}$ wide and $190 \mathrm{~mm}$ long. The height of the center of the bar is the same height of the subject's eyes. Five male students were examined as subjects. We measured the movements of the $\mathrm{C}$. G. of the subject with two kinds of examination.

In the first (experiment I), the bar swings at $f_{1} \mathrm{~Hz}$ in front of him with the height of his waist as a center of rotation and the equilibrium line of the bar vibration deviates gradually right or left at $f_{2} \mathrm{~Hz}$. The angle $\theta$ of the bar are expressed by

$$
\theta=\theta_{1} \sin 2 \pi f_{1} t+\theta_{2} \sin 2 \pi f_{2} t,
$$

where the vibration frequency $f_{1}$ is $0.2,0.25,0.3$ or $0.4 \mathrm{~Hz}$ and the deviation frequency $f_{2}$ is $0.002,0.004$ or $0.008 \mathrm{~Hz}$. In the second, the C. G. movements

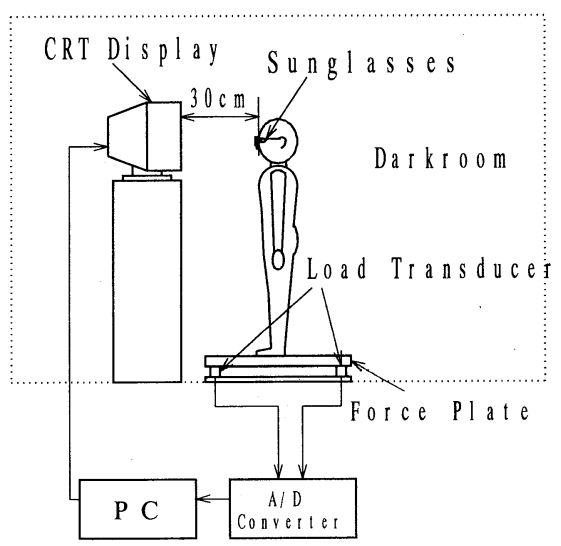

Fig. 1 Experimental apparatus.

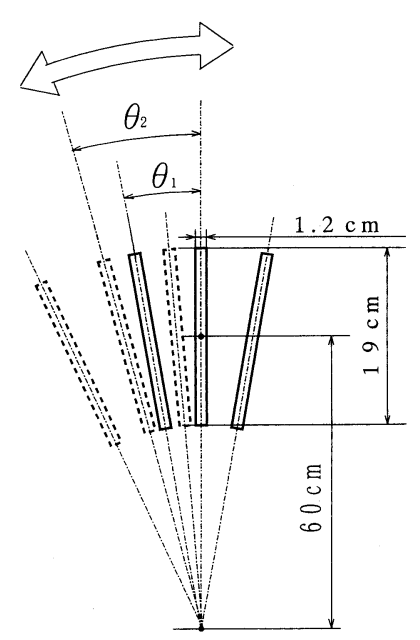

Fig. 2 Swinging pattern.

were measured for $11 \mathrm{~min}$ to observe the effect of gazing for a long time (experiment II). In this case, we set $\theta_{2}=0$.

\section{EXPERIMENTAL RESULTS AND DISCUSSIONS}

3.1 C. G. Movements in Deviation of the Equilibrium Line

C. G. Movements of a subject were measured when he was gazing at a swinging bar made with a personal computer. At first, the movements were measured when a subject was standing in a dark room without any picture. The locus of C. G. projected on the force plate is shown in Fig. 3(a). At the next, the movements were measured when he was standing in a light place in order to examine eye 


\section{H. TAKADA and M. OHBA : POSTURAL STABILITY OF HUMAN BODY GAZING AT PICTURES}

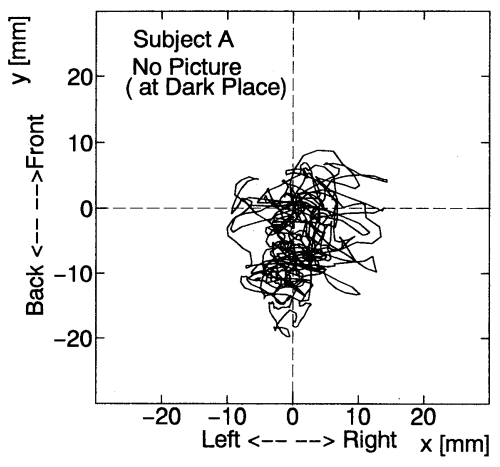

Fig. 3(a) Locus of C. G. in a dark room without any picture.

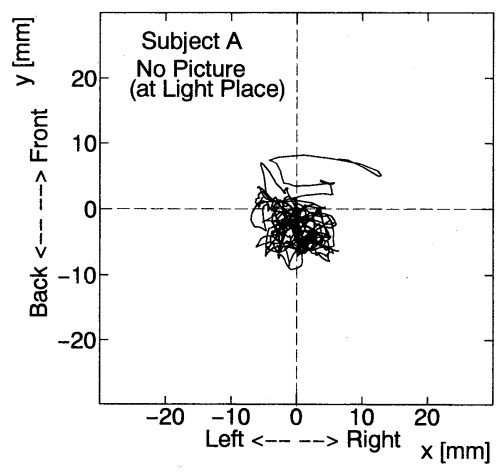

Fig. 3(b) Locus of C. G. at a light place.

information. The locus is shown in Fig. 3(b). Apparently, we can stand more stably in a light place than in a dark place.

Next, we measured only the right and left directions of the movements of C. G. to examine the effect of the bar swinging and the equilibrium line deviation. When the equilibrium line of the swinging bar deviates right or left, we examined how we could stand. Figure 4 shows the locus of C. G. when the equilibrium line deviates right. You can see the average position of C. G. deviates right. The coverage of the locus in Fig. 4 is smaller than that in Fig. 3(a). This means gazing may makes our posture more stable even when the equilibrium line deviates. The locus of C. G. is shown in Fig. 5 when the equilibrium line deviates left. You can see the average position also deviates left. This means we can sway in the same direction as the equilibrium line deviates.

Figure 6 shows the movements of C. G. with respect to time. The equilibrium line deviates right

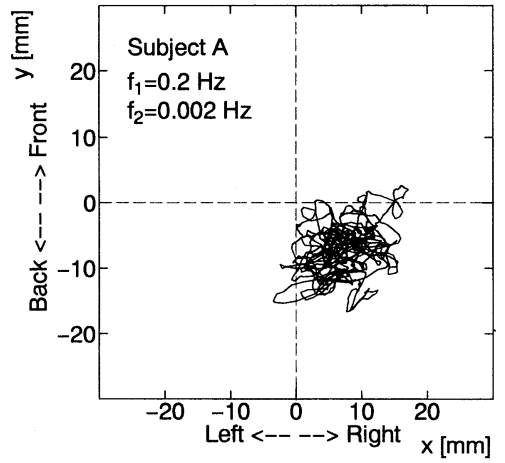

Fig. 4 Locus of C. G. when the equilibrium line deviates right.

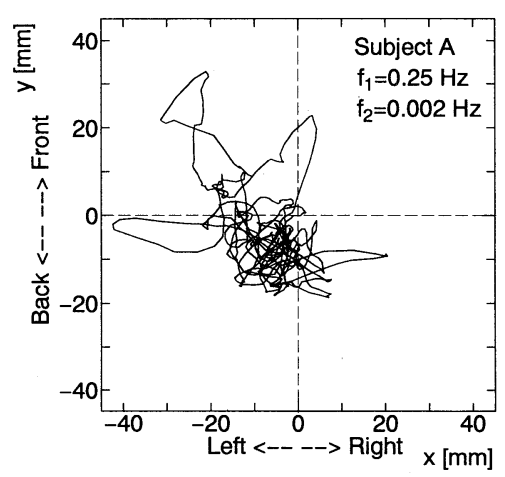

Fig. 5 Locus of C. G. when the equilibrium line deviates left.

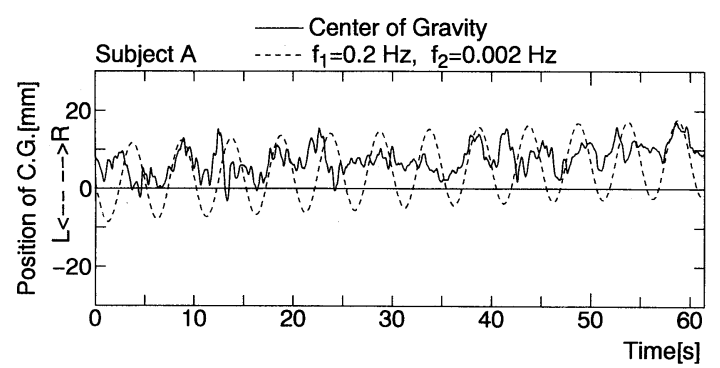

Fig. 6 Movements of C. G. when the equilibrium line deviates right.

and so the position of C. G. also deviates gradually in the same direction. From this wave you can see the position not only deviates but also swings at the similar frequency to the bar frequency, for example between 7 and $12 \mathrm{~s}, 17$ and $20 \mathrm{~s}$ and so on. From the viewpoint, we stand tuning to the bar sway. Figure 7 shows the C. G. movements in case of the left deviation of the equilibrium line. This case 


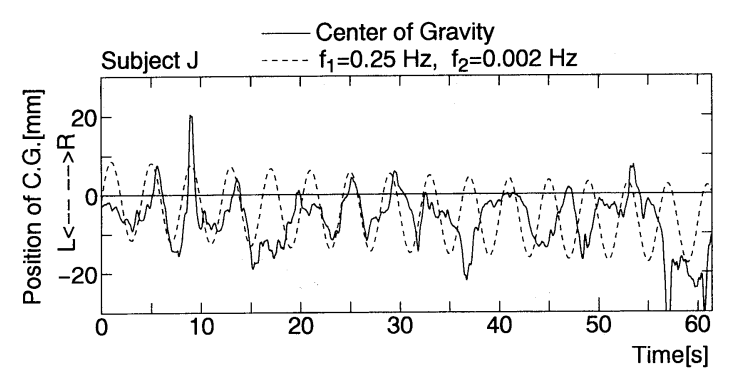

Fig. 7 Movements of C. G. when the equilibrium line deviates left.

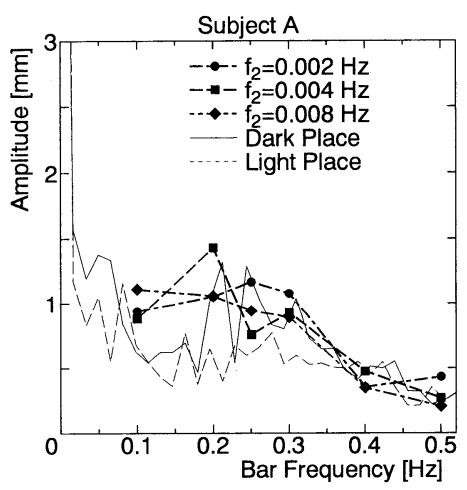

Fig. 8 Frequency responses.

also indicates he swings at the similar frequency to that of the bar sway and at the same time his C. G. deviates gradually left. The frequency responses are shown in Fig. 8. We have the peak between at 0.2 and $0.3 \mathrm{~Hz}$, that is, our standing posture has the resonance frequency between 0.2 and $0.3 \mathrm{~Hz}$.

\subsection{G. Movements in Gazing for a Long Time}

We measured the movements of C. G. when we stand gazing at a moving picture for a long time. As an index of unstability of human posture, we considered the next : the index like the variance of subject's C. G. for the total time (11 min), the index like the variance of that for every minute and the total distance of the locus where the C. G. moved. As a result, we decided the index like the variance of the subject's C. G. for every minute could indicate the unstability of the posture best among three indices for a long time (11 $\mathrm{min})$.

Figure 9 shows the degree of sway of C. G. when a subject stands for $11 \mathrm{~min}$ in a darkroom. The values were calculated by

$$
S=\sum_{i=1}^{N}\left(x_{i}-\bar{x}\right)^{2}+\left(y_{i}-\bar{y}\right)^{2},
$$

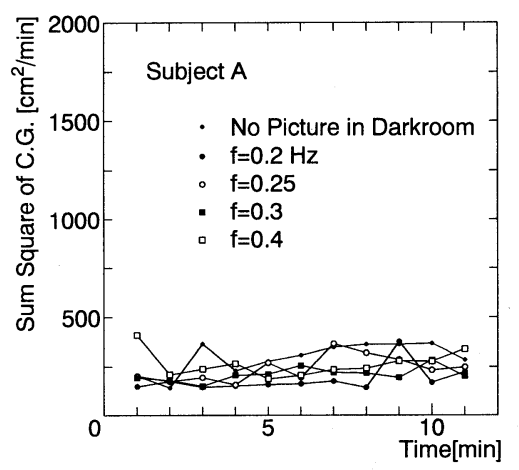

Fig. 9 Degree of sway in gazing for a long time (Subject A).

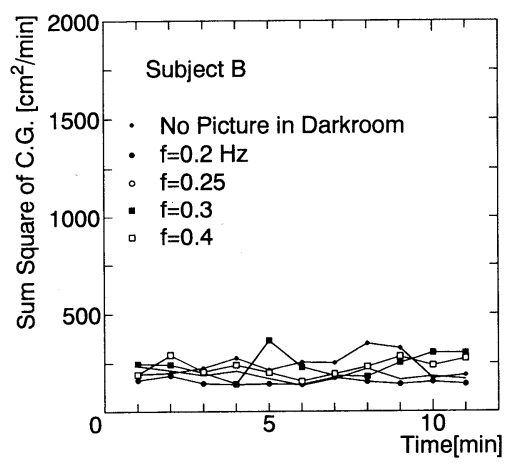

Fig. 10 Degree of sway in gazing for a long time (Subject C).

where, the $x_{i}$ and $y_{i}$ are the position of C. G. when sampled. The $\bar{x}$ and $\bar{y}$ are the averages for $1 \mathrm{~min}$. For example, the value in $5 \mathrm{~min}$ in Figure indicates the value between 4 and $5 \mathrm{~min}$. The $N$ equals 600 because the sampling time is $0.1 \mathrm{~s}$. This $S$ indicates the unstability of a subject's posture. From this figure this subject is hardly affected by a swinging bar for $11 \mathrm{~min}$, but we can see he gets tired a little in $11 \mathrm{~min}$. The results obtained from the subject $\mathrm{B}$ are similar to those from the subject A.

Figure 10 shows the results of subject $\mathrm{C}$. He violently swings in a whole time, but he can stand a little more stably when he is gazing at a swinging bar than when he is gazing at no picture in a darkroom. The results from the subject $\mathrm{D}$ and the subject $\mathrm{E}$ are similar to those from the subject $\mathrm{C}$. We can find some people are affected by a swinging bar and can stand more stably in gazing at one.

Next, we plotted the component of $0.2,0.3$ and 0.4 $\mathrm{Hz}$ from a subject's sway when the bar frequency is $0.2,0.3$ and $0.4 \mathrm{~Hz}$ respectively. This means when 


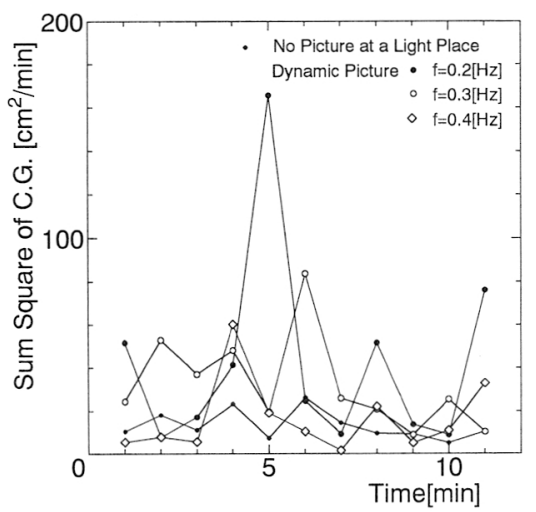

Fig. $110.2,0.3$ and $0.4 \mathrm{~Hz}$ component of sway (Subject A).

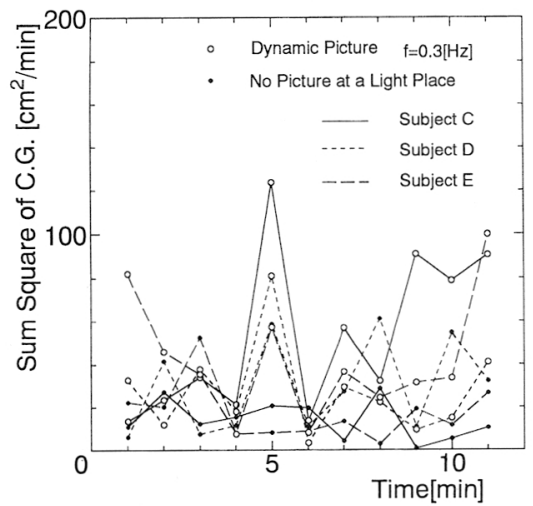

Fig. $120.3 \mathrm{~Hz}$ component of sway (Subject C, $\mathrm{D}$ and $\mathrm{E})$.

and how his C. G. sway is tuned to the bar sway. The results of his sway when he stands at a light place are shown in the same figure to refer the individuality. The $0.2,0.3$ and $0.4 \mathrm{~Hz}$ sway components of the subject A are shown in Fig. 11. He swings at $0.2 \mathrm{~Hz}$ in $6 \mathrm{~min}$ even at a light place, but he swings in $5 \mathrm{~min}$ more violently when he is gazing at a $0.2 \mathrm{~Hz}$ swinging bar than when he stands at a light place. In the case of $0.3 \mathrm{~Hz}$, he swings at this frequency more violently except one point in gazing in the swinging bar than in standing at a light place. In the case of $0.4 \mathrm{~Hz}$, he swings more violently in gazing at the swinging bar, but the values are smaller at the both lines than at $0.2 \mathrm{~Hz}$ and at $0.3 \mathrm{~Hz}$. Other subjects were examined at $0.3 \mathrm{~Hz}$ swinging bar because we are affected most strongly at $0.3 \mathrm{~Hz}$. The results of subject $\mathrm{B}$ are similar to subject $\mathrm{A}$, and so there is no figure in this paper. The results of subject $\mathrm{C}, \mathrm{D}$ and $\mathrm{E}$ are shown in Fig. 12. The subject $\mathrm{C}$ tunes to $0.3 \mathrm{~Hz}$ swinging bar in $5 \mathrm{~min}$ and at the last time. The subject $\mathrm{D}$ swings violently in $5 \mathrm{~min}$, but he swings in $5 \mathrm{~min}$ even at a light place, and so we think he is hardly affected by a swinging bar. The subject $\mathrm{E}$ is affected at the first $1 \mathrm{~min}, 5$ min and the last $1 \mathrm{~min}$. That means he tunes to the swing bar at the first time, and his posture becomes gradually stable. In 4 min, he can stand most stably, nevertheless he tunes in $5 \mathrm{~min}$. All five subjects tune to the swinging bar in $5 \mathrm{~min}$. This time may be the limit we can control our postures when we are gazing at a swinging bar in a darkroom.

\section{CONCLUSIONS}

We conclude the next as a result we measured movements of 5 subject's center of gravity when they stand gazing at a swinging picture in a darkroom.

1. When the equilibrium line of the swinging bar deviates right or left, the average positions of center of gravity also deviates in the same direction.

2. All subjects we tested tune to the swinging bar in $5 \mathrm{~min}$ after they start their examinations. This time may be the limit we can control our postures when we are gazing at a swinging bar in a darkroom.

\section{REFERENCES}

1) M. J. Griffin, "Levels of whole-body vibration affecting human vision," Aviat. Space Environ. Med., August, 1033-1040 (1975).

2) M. J. Griffin, "Eye motion during whole-body vertical vibration," Hum. Factors 18, 601-606 (1976).

3) A. J. Benson and G. R. Barnes, "Vision during angular oscillation: The dynamic interaction of visual and vestibular mechanisms," Aviat. Space Environ. Med., January, 340-345 (1978).

4) K. Taguchi, M. Kikukawa, and T. Ishiyama, "The effects of optokinetic stimulation on the head movement," Equilibrium Res. 40, 242-250 (1981) (in Japanese).

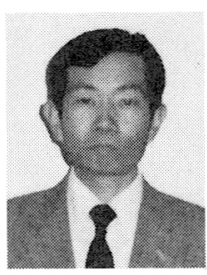

Hajime Takada received the B. E., M. E. and Dr. Eng. degrees from the University of Tokyo in 1977, 1979 and 1982, respectively. He was a lecturer, an Associate Professor of Department of Mechanical Engineering at Faculty of Engineering, Yokohama National University from 1982 and from 1985 respectively. He is now a Professor of Mechanical Engineering and Materials Science at Graduate School of Engineering, 
Yokohama National University. $\mathrm{He}$ is a member of Japan Society of Mechanical Engineers and Japan Society of Ergonomics.

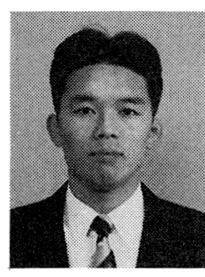

Motoki Ohba received the B. E. and M. E. degrees from Yokohama National University in 1994 and 1996, respectively. He is now an engineer of Komatsu Co. Ltd. 